in a way useable by those whose job it is to solve practical problems. In the real situation it is better to be master of a little science rather than the slave of much. The approximate methods of the navigator are far more likely to be useful in crime investigation than the elegant solutions of the mathematician.

ACKNOWLEDGEMENTS. The credit for any potential usefulness, or for any apparent originality, of the views expressed here, is largely due to the professional competence, friendship and stimulating company of the police colleagues named earlier in this article. The inadequacies are entirely the writer's.

\title{
Position Fixing with Neither Dead Reckoning nor GMT
}

\author{
W. B. Fu \\ (Hong Kong Polytechnic)
}

This is a short summary of a paper which shows what a navigator can do to fix his position at sea using basic equipment (sextant, hand-held compass, almanac, four-figure mathematical tables) without the help of dead reckoning or the benefits of GMT.

To proceed from an almost complete absence of spatial and temporal data the navigator has to observe the sextant altitudes $\left(A_{1}, A_{2}, A_{3}\right)$ of two stars and the Moon, respectively. Using the measurements $\left(A_{1}, A_{2}\right)$ of the stars and data provided by the almanac on their declinations $\left(D_{1}, D_{2}\right)$ and sidereal hour angles $\left(S_{1}, S_{2}\right)$ the navigator can calculate his latitude $(L)$ by the iterative solution of an equation derivable from spherical trigonometry:

where

$$
\arccos \left(H_{1}\right)+\arccos \left(H_{2}\right)-S_{1}+S_{2}=0
$$

$$
H_{1}=\frac{\sin A_{1}-\sin L \sin D_{i}}{\cos L \cos D_{1}} \quad(i=1,2)
$$

From this value of the latitude he can deduce the local hour angle of Aries. He then combines these calculated results with the lunar altitude $\left(A_{3}\right)$ to determine the GMT by solving iteratively another equation from spherical trigonometry:

$$
\sin A_{3}-\sin L \sin D_{3}-\cos L \cos D_{3} \cos H_{3}=0
$$

Here $D_{3}$ and $H_{3}$, which are time-dependent quantities, denote respectively the declination and local hour angle of the Moon.

The GMT having been found, the navigator can easily deduce his longitude. With care the results for both the latitude and longitude can be obtained to the nearest minute of arc. The penalty for the almost complete ignorance of one's position and time is a great deal of numerical work which may be too demanding for navigators with only an O-level in mathematics.

The present method is different in principle from the now obsolete lunar distance method which requires the measurements of the altitude of the Moon and one star, and the angular distance between them.

Readers who are interested in this subject are invited to write to the author, care of the RIN for details. 